\title{
Human Parechovirus Meningoencephalitis: Neuroimaging in the Era of Polymerase Chain Reaction-Based Testing
}

\author{
(D) A. Sarma, DE. Hanzlik, Dr. Krishnasarma, DL. Pagano, and (D) Pruthi
}

\begin{abstract}
SUMMARY: Human parechovirus infection is an increasingly recognized cause of neonatal meningoencephalitis. We describe characteristic clinical features and brain MR imaging abnormalities of human parechovirus meningoencephalitis in 6 infants. When corroborated by increasingly available polymerase chain reaction-based testing of the CSF, the distinctive MR imaging appearance may yield a specific diagnosis that obviates costly and time-consuming further clinical evaluation. In our study, infants with human parechovirus presented in the first 35 days of life with seizures, irritability, and sepsis. MR imaging consistently demonstrated low diffusivity within the thalami, corpus callosum, and subcortical white matter with a frontoparietal predominance. $\mathrm{Tl}$ and $\mathrm{T} 2$ shortening connoting white matter injury along the deep medullary veins suggests venous ischemia as an alternative potential pathogenetic mechanism to direct neuroaxonal injury.
\end{abstract}

ABBREVIATIONS: $\mathrm{HPeV}=$ human parechovirus; $\mathrm{PCR}=$ polymerase chain reaction

$\mathrm{H}$ istorically, many cases of neonatal meningoencephalitis have had no specific identifiable cause. ${ }^{1-10}$ Human parechovirus $(\mathrm{HPeV})$ is an increasingly recognized cause of neonatal meningoencephalitis in the era of rapid polymerase chain reaction (PCR)based assays for $\mathrm{CSF}^{7} \mathrm{HPeV}$ is a single-stranded ribonucleic acid virus from the family Picornaviridae. Among 19 identified genotypes, $\mathrm{HPeV}-3$ is most commonly identified in severe infection of young infants and CNS infection. ${ }^{10,11}$ Infection occurs via a respiratory or gastrointestinal route with hematogenous dissemination. ${ }^{10}$ Cases usually occur with seasonal outbreaks in summer and autumn with biannual cycles. ${ }^{10}$ Common reported clinical manifestations in minor infection include gastroenteritis and respiratory symptoms. Similar to enterovirus, typical clinical presentation in young infants with severe CNS infection includes fever, rash, irritability, and features of sepsis with rare CSF pleocytosis. Children younger than 2 years of age are at greatest

Received March 29, 2019; accepted after revision June 2.

From the University Medical Center (A.S., E.H., L.P., S.P.), Vanderbilt University, Nashville, Tennessee; and Boston Children's Hospital (R.K.), Harvard Medical School, Boston, Massachusetts.

Data previously presented in a different format for oral presentation at: Society for Pediatric Radiology Annual Meeting and Categorical Course, April 30 to May 4, 2019; San Francisco, California.

Please address correspondence to Sumit Pruthi, MD, Vanderbilt University Medical Center, Vanderbilt University, Department of Radiology \& Radiological Sciences,

Monroe Carell Jr. Children's Hospital, 2200 Children's Way, Nashville, TN 37232;

e-mail: sumit.pruthi@vumc.org; @sumsnet

三 Indicates article with supplemental on-line table.

http://dx.doi.org/10.3174/ajnr.A6118 risk, and illness is especially severe in children younger than 6 months. ${ }^{10}$

Because of its noninflammatory CSF profile, $\mathrm{HPeV}$ meningoencephalitis has likely been under-recognized. Indeed, since the recent advent of PCR-based assays, $\mathrm{HPeV}$ has been identified as the second most common causative pathogen after enterovirus in infants younger than 2 months of age. ${ }^{10}$ Multiple case reports and small case series suggest a distinctive clinical and imaging presentation. ${ }^{12-15}$ However, few descriptions exist within the radiology literature. ${ }^{12}$ This report provides detailed clinical and radiologic description of 6 infants with $\mathrm{HPeV}$ meningoencephalitis with a current literature review.

\section{Case Series}

No institutional review board approval was required for this retrospective series. Six cases were identified between October 2016 and September 2018 at Monroe Carell Jr. Children's Hospital at Vanderbilt and Boston Children's Hospital.

Electronic medical record review yielded birth history and gestational age, age at presentation, presenting symptoms, length of hospital and intensive care unit stay, CSF analysis (cell count, glucose, protein, viral PCR analysis, FilmArray meningoencephalitis panel, BioFire Diagnostics, Salt Lake City, Utah; and qualitative PCR assay for HPeV, ARUP Laboratories, Salt Lake City, Utah), electroencephalography reports and descriptions of seizures, and the status of the patient at discharge and follow-up.

All patients underwent brain MR imaging. Two of 6 underwent sonography, and 2 underwent CT before MR imaging. The MR imaging technique varied by institution. All patients at Mon- 
roe Carell Jr. Children's Hospital at Vanderbilt were scanned on a 1.5T MR imaging unit (Phillips Achieva 3T, Phillips Healthcare, Best, the Netherlands), and all patients at Boston Children's Hospital were scanned on a 3T MR imaging unit (Siemens Skyra 3T, Siemens, Erlangen, Germany). Sequences included 3D T1weighted MPRAGE, T2-weighted DWI, gradient recalled-echo (3/6), SWI (3/6), postgadolinium T1-weighted (1/6), time-offlight MRA (1/6), time-of-flight MRV (2/6), and multivoxel MR spectroscopy (3/6). Three-month follow-up MR imaging was performed in $1 / 6$. All MR imaging studies were retrospectively reviewed by 2 fellowship-trained pediatric neuroradiologists.

Clinical data are summarized in the On-line Table. Six patients presented from 6 to 35 days of life with irritability $(n=4)$, poor feeding $(n=5 / 6)$, and fever $(n=6 / 6)$. Multifocal seizures were

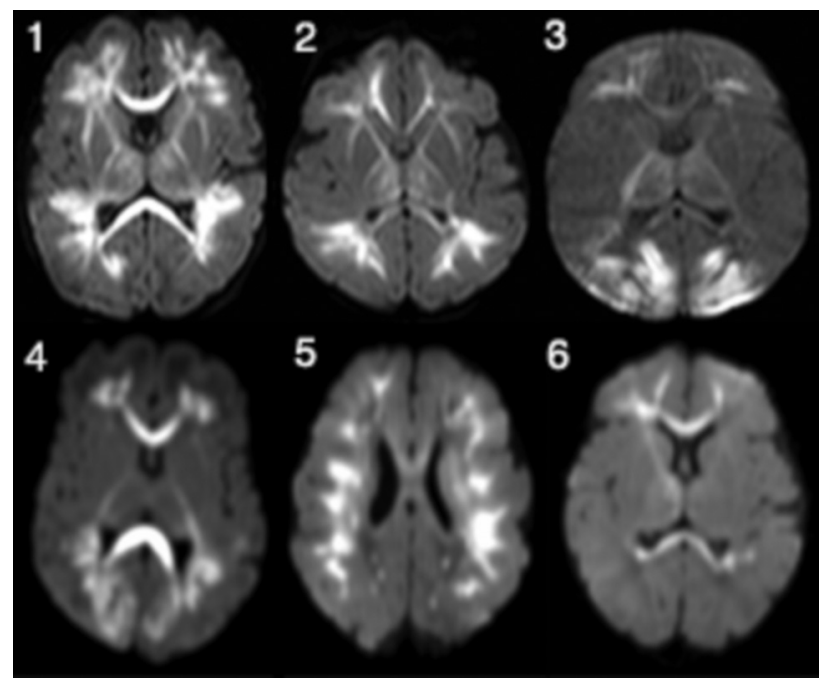

FIG 1. Axial diffusion-weighted images in patients 1-6 (infants with $\mathrm{HPeV}$ infection) demonstrate low diffusivity variably involving the periventricular white matter with frontoparietal predominance and also involving the corpus callosum, thalami, and internal and external capsules. Patients 3 and 4 exhibit greater involvement of the occipital white matter and the occipital cortex. Patient 5 shows more confluent involvement of the subcortical white matter. present in 5/6. Four full-term neonates presented in the first 10 days of life, and 2 premature neonates ( 33 and 35 weeks' gestational age) presented on days of life 20 and 35. CSF showed no pleocytosis and was culture negative for bacterial pathogens and $\mathrm{HSV}$ with PCR-based testing positive for $\mathrm{HPeV}$ in 5/6. In 1 case, $\mathrm{HPeV}$ was detected in serum and stool samples.

Head sonography in 2/6 and head CT in 1/6 had normal findings. Head CT in 1/6 showed parieto-occipital hypoattenuation and loss of gray-white differentiation.

The most characteristic finding in all cases was diffuse/multifocal frontal-predominant subcortical white matter and callosal involvement with associated low diffusivity (Fig 1). The thalami were involved in all cases to varying degrees. The $2 / 6$ with striking occipital involvement were also the only cases with cortical signal abnormality (also in the occipital lobe). No cases exhibited signal abnormality within the basal ganglia, hippocampi, brain stem, and cerebellum. Branching T1 and T2 shortening along the distribution of the deep medullary veins was noted in 4/6 (Fig 2). In $2 / 6$, these foci were identified on either T1- or T2-weighted imaging but not both. One patient (patient 4, Fig 2) had mild diffuse white matter T2 prolongation, even considering the premature myelination pattern. Scant susceptibility foci consistent with hemorrhage or mineralization were noted in the periatrial white matter in $1 / 6$. Of the $3 / 6$ who underwent MR spectroscopy, only one had elevated lactate in the basal ganglia. Postcontrast, MRA, and MRV results were normal.

\section{DISCUSSION}

This series describes 6 infants with HPeV meningoencephalitis and characteristic MR imaging findings that rapidly yielded a diagnosis in conjunction with PCR testing of the CSF.

Distinctive supratentorial white matter and callosal diffusion abnormality on MR imaging is shared between ours and other published series. ${ }^{3,12,13,15-17}$ Thalamic involvement and sparing of the posterior fossa structures have been reported variably but were universally present in our cases. ${ }^{12,18} \mathrm{~T} 1$ - and T2-shortening foci branching along the distribution of the deep medullary veins

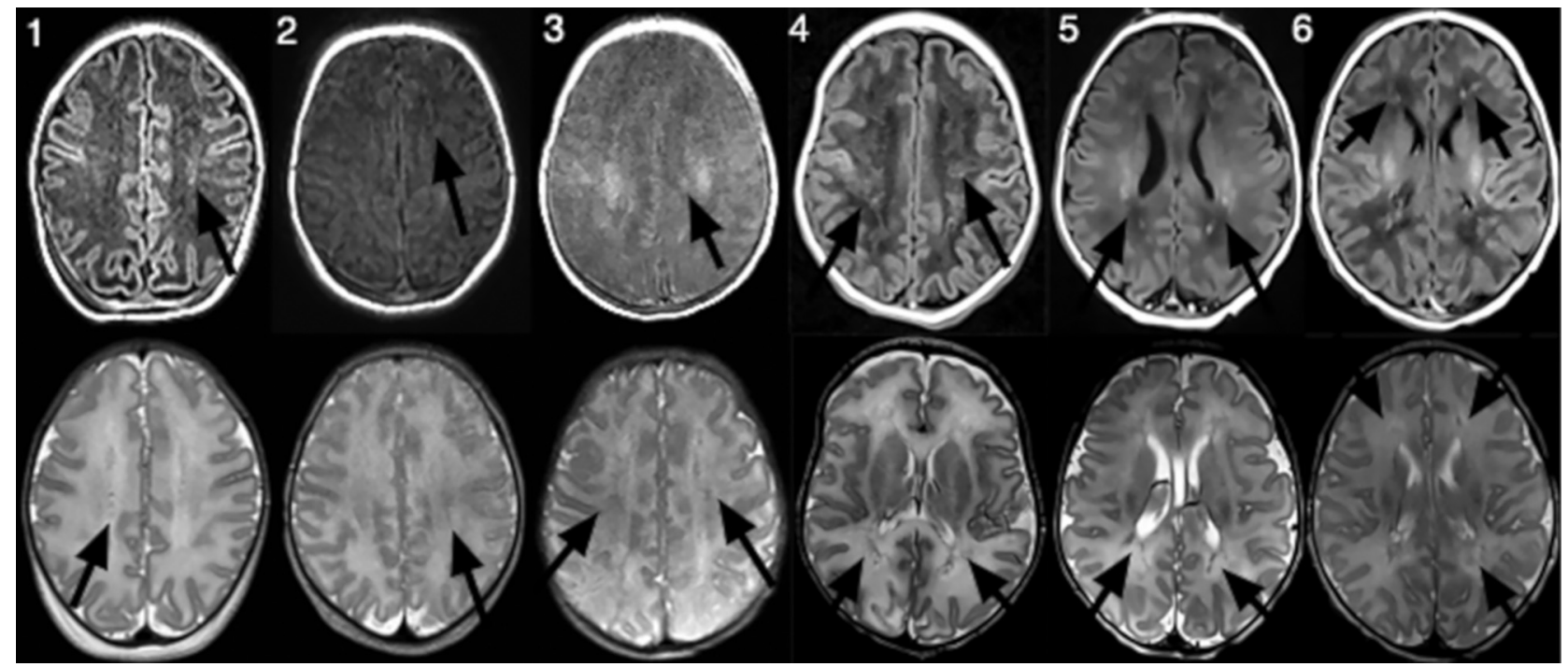

FIG 2. Axial 3D T1-weighted MPRAGE images (upper row) and axial T2-weighted images (lower row) in patients $1-6$ (infants with HPeV infection) demonstrate foci of $\mathrm{T} 1$ and/or $\mathrm{T} 2$ shortening following the distribution of the deep medullary veins (arrows). 
is consistent with examples presented by Belcastro et $\mathrm{al}^{13}$ and Verboon-Maciolek et $\mathrm{al}^{9}$ but has not been consistent across studies. Occipital white matter and cortical involvement in $2 / 6$ cases is also dissimilar to previous descriptions and may be explained by hypoglycemia in one and transient hypoglycemia or, alternatively, a seizure-related or vascular phenomenon such as posterior reversible encephalopathy syndrome in the other.

The pathophysiology of CNS injury in $\mathrm{HPeV}$ infection is not well-understood. The pattern of MR imaging signal abnormality preferentially affecting the supratentorial white matter tracts in our cases could suggest neuroaxonal tropism; however, signal abnormality along the course of the deep medullary veins on T1and T2-weighted imaging in 5/6 cases suggests perivenular invasion or venous ischemia as a potential alternative etiology. Supporting the first hypothesis, a study of patients with clinical CNS infection and stool samples positive for HPeV-3 showed faster replication in a cultured neural cell line. ${ }^{19}$ Verboon-Maciolek et $\mathrm{al}^{9}$ and $\mathrm{Volpe}^{20}$ reported that the HPeV RNA activates toll-like receptors in microglia, resulting in the release of inflammatory substances that injure preoligodendrocytes and axons as part of an innate immune response, as well as toll-like receptors in neurons and growing axons, resulting in direct injury. Alternatively, supporting the venous ischemia hypothesis, a postmortem examination study of 2 premature neonates (33 and 34 weeks gestational age) with fatal $\mathrm{HPeV}$ infection by Bissel et al ${ }^{11}$ demonstrated severe inflammatory changes of the periventricular white matter with macrophage infiltration and astrocytosis. While HPeV-3 RNA probes hybridized with abundant viral particles within brain meningeal cells and blood vessel walls, they did not reveal the presence of $\mathrm{HPeV}$ within parenchymal cells. The authors therefore hypothesized that parenchymal injury is a consequence of vascular compromise rather than direct infection. Low diffusivity of the corpus callosum may result from seizure activity and/or Wallerian degeneration, given the relative resistance of the corpus callosum to ischemic insults. Low diffusivity in the thalamus may be due to some combination of venous ischemia and seizure activity.

While the clinical and imaging characteristics of $\mathrm{HPeV}$ meningoencephalitis are characteristic in a neonate with sepsis and seizures, there is a differential diagnosis. Distinction from enterovirus, rotavirus, and chikungunya infection may not be possible. ${ }^{21,22}$ In contradistinction to neonates with hypoxic-ischemic injury, neonates with $\mathrm{HPeV}$ infection lack a history of a sentinel event. Frontoparietal white matter-predominant low diffusivity and sparing of the basal ganglia favor $\mathrm{HPeV}$ encephalitis over hypoxic-ischemic injury. ${ }^{12}$ Isolated sulfite oxide deficiency and molybdenum cofactor deficiency can present in the neonatal period; however, the basal ganglia are usually affected.

Reported long-term outcomes are variable, with limited available data, but overall suggest adverse developmental outcomes in patients with encephalitis and abnormal MR imaging findings. In a study comparing longitudinal gross motor development between infants with $\mathrm{HPeV}$ infection and those with no pathogen found, a statistically significant difference was noted at 6 months, but not at 24 months. ${ }^{23}$ In a study in which 6 patients with clinically diagnosed $\mathrm{HPeV}$ encephalitis underwent MR imaging during both acute illness and follow-up developmental assessment at
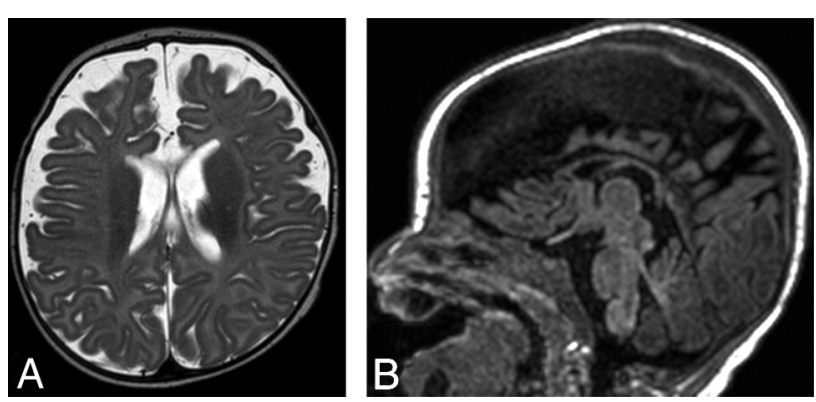

FIG 3. Axial T2-weighted image $(A)$ and sagittal T1-weighted image $(B)$ obtained in patient 1 approximately 3 months after acute $\mathrm{HPeV}$ infection show new diffuse enlargement of the extra-axial spaces and thinning of the corpus callosum, suggesting volume loss.

12 months, Britton et $\mathrm{al}^{15}$ reported concern for abnormal development in all 6. Verboon-Maciolek et $\mathrm{al}^{9}$ found that in the $50 \%$ (5/10) of patients in their cohort who had a developmental disability, the severity of imaging abnormalities correlated with neurodevelopmental outcome. Two patients in our cohort have been noted to be clinically well at 6- to 8-week follow-up; however, long-term follow-up data are not yet available.

A few reports have described long-term imaging follow-up, with mixed results. Pariani et $\mathrm{al}^{24}$ and Amarnath et $\mathrm{al}^{12}$ had resolution of imaging abnormalities at 2-month and 1-year follow-up, respectively. In 3 patients, Verboon-Maciolek et $\mathrm{al}^{9}$ reported variable degrees of visible white matter injury at 3-month-to-7-year follow-up, with abnormalities ranging from mild periventricular gliosis to extensive white matter volume loss and cystic encephalomalacia. Brownell et al ${ }^{16}$ described a patient with severe white matter injury at day 3 , with only mild white matter signal abnormality and callosal thinning at 1 year. On the contrary, Belcastro et $\mathrm{al}^{13}$ described the development of extensive cystic leukomalacia at 4-month follow-up in 1 patient. Some data suggest that preterm infants are at higher risk for severe cystic leukoencephalomalacia. ${ }^{9-11}$ One patient in our study had a 3-month follow-up MR imaging showing diffuse parenchymal volume loss (Fig 3). Further study will help to correlate the severity of clinical and imaging findings with long-term outcomes.

Current study limitations include the retrospective design and small number of cases. Viral genotyping was not performed, and this study did not determine imaging characteristics that distinguish among viral genotypes. Furthermore, imaging techniques varied. Referral to imaging may not be performed in less severe cases, biasing MR imaging requests toward patients with severe clinical presentation. An additional limitation is limited clinical and imaging follow-up, which warrants further study. Finally, findings in patients with less severe disease are currently not wellestablished and could also be determined by future study.

The stereotypical MR imaging appearance correlated with molecular testing in our patients yielded a specific diagnosis of $\mathrm{HPeV}$ meningoencephalitis, resulting in a less extensive metabolic and genetic work-up than would have been undertaken otherwise. Knowledge of the distinctive imaging findings in young infants with $\mathrm{HPeV}$ meningoencephalitis thus has potential for substantial cost- and time-savings. 


\section{CONCLUSIONS}

$\mathrm{HPeV}$ infection is an increasingly recognized cause of neonatal sepsis or meningoencephalitis, often presenting with seizures, and MR imaging is an essential component of the clinical evaluation of seizures in neonates. DWI is of paramount importance in making the diagnosis and shows distinctive low diffusivity of the supratentorial white matter with frontoparietal predominance, with involvement of the corpus callosum and thalami and sparing of the basal ganglia, brain stem, and cerebellum.

Disclosures: Asha Sarma—UNRELATED: Royalties: Cambridge University Press, Comments: textbook previously published on a different topic.

\section{REFERENCES}

1. Leber AL, Everhart K, Balada-Llasat JM, et al. Multicenter evaluation of BioFire FilmArray Meningitis/Encephalitis Panel for detection of bacteria, viruses, and yeast in cerebrospinal fluid specimens. J Clin Microbiol 2016;54:2251-61 CrossRef Medline

2. Midgley CM, Jackson MA, Selvarangan R, et al. Severe parechovirus 3 infections in young infants: Kansas and Missouri, 2014. J Pediatric Infect Dis Soc 2018;7:104-12 CrossRef Medline

3. Khatami A, McMullan BJ, Webber M, et al. Sepsis-like disease in infants due to human parechovirus type 3 during an outbreak in Australia. Clin Infect Dis 2015;60:228-36 CrossRef Medline

4. Ferreras Antolín L, Kadambari S, Braccio S, et al. Increased detection of human parechovirus infection in infants in England during 2016: epidemiology and clinical characteristics. Arch Dis Child 2018;103:1061-66 CrossRef Medline

5. Vergnano S, Kadambari S, Whalley K, et al. Characteristics and outcomes of human parechovirus infection in infants (2008-2012). Eur J Pediatr 2015;174:919-24 CrossRef Medline

6. Sharp J, Harrison CJ, Puckett K, et al. Characteristics of young infants in whom human parechovirus, enterovirus or neither were detected in cerebrospinal fluid during sepsis evaluations. Pediatr Infect Dis J 2013;32:213-16 CrossRef Medline

7. Aizawa $Y$, Izumita R, Saitoh A. Human parechovirus type 3 infection: an emerging infection in neonates and young infants. J Infect Chemother 2017;23:419-26 CrossRef Medline

8. Joseph L, May M, Thomas M, et al. Human parechovirus 3 in infants: expanding our knowledge of adverse outcomes. Pediatr Infect Dis J 2019;38:1-5 CrossRef Medline

9. Verboon-Maciolek MA, Groenendaal F, Hahn CD, et al. Human parechovirus causes encephalitis with white matter injury in neonates. Ann Neurol 2008;64:266-73 CrossRef Medline

10. Olijve L, Jennings L, Walls T. Human parechovirus: an increasingly recognized cause of sepsis-like illness in young infants. Clin Microbiol Rev 2018;31 CrossRef Medline

11. Bissel SJ, Auer RN, Chiang CH, et al. Human parechovirus 3 menin- gitis and fatal leukoencephalopathy. J Neuropathol Exp Neurol 2015; 74:767-77 CrossRef Medline

12. Amarnath C, Helen Mary T, Periakarupan A, et al. Neonatal parechovirus leucoencephalitis: radiological pattern mimicking hypoxicischemic encephalopathy. Eur J Radiol 2016;85:428-34 CrossRef Medline

13. Belcastro V, Bini P, Barachetti R, et al. Teaching neuro images: neonatal parechovirus encephalitis: typical MRI findings. Neurology 2014;82:e23 CrossRef Medline

14. de Jong EP, Holscher HC, Steggerda SJ, et al. Cerebral imaging and neurodevelopmental outcome after entero- and human parechovirus sepsis in young infants. Eur J Pediatr 2017;176:1595-602 CrossRef Medline

15. Britton PN, Dale RC, Nissen MD, et al; PAEDS-ACE Investigators. Parechovirus encephalitis and neurodevelopmental outcomes. $\mathrm{Pe}$ diatrics 2016;137:e20152848 CrossRef Medline

16. Brownell AD, Reynolds TQ, Livingston B, et al. Human parechovirus-3 encephalitis in two neonates: acute and follow-up magnetic resonance imaging and evaluation of central nervous system markers of inflammation. Pediatr Neurol 2015;52:245-49 CrossRef Medline

17. Leow JY, Gupta R, Sohal AP. Human parechovirus central nervous system infection: a rare cause of neonatal encephalitis. J Paediatr Child Health 2015;51:1244 CrossRef Medline

18. Van Zwol AL, Lequin M, Aarts-Tesselaar C, et al. Fatal neonatal parechovirus encephalitis. BMJ Case Rep 2009;2009 CrossRef Medline

19. Westerhuis BM, Koen G, Wildenbeest JG, et al. Specific cell tropism and neutralization of human parechovirus types 1 and 3: implications for pathogenesis and therapy development. J Gen Virol 2012; 93(Pt 11):2363-70 CrossRef Medline

20. Volpe JJ. Neonatal encephalitis and white matter injury: more than just inflammation? Ann Neurol 2008;64:232-36 CrossRef Medline

21. Verboon-Maciolek MA, Truttmann AC, Groenendaal F, et al. Development of cystic periventricular leukomalacia in newborn infants after rotavirus infection. $J$ Pediatr 2012;160:165-68.e1 CrossRef Medline

22. Ganesan K, Diwan A, Shankar SK, et al. Chikungunya encephalomyeloradiculitis: report of 2 cases with neuroimaging and 1 case with autopsy findings. AJNR Am J Neuroradiol 2008;29:1636-37 CrossRef Medline

23. van Hinsbergh TM, Elbers RG, van Furth MA, et al. Longitudinal association between human parechovirus central nervous system infection and gross-motor neurodevelopment in young children. Pediatr Infect Dis J 2019;38:110-14 CrossRef Medline

24. Pariani E, Pellegrinelli L, Pugni L, et al. Two cases of neonatal human parechovirus 3 encephalitis. Pediatr Infect Dis J 2014;33:1191-93 CrossRef Medline 